\title{
Exploring relationships between students' interaction and learning with a haptic virtual biomolecular model
}

Konrad J. Schönborn, Petter Bivall and Lena A. E. Tibell

\section{Linköping University Post Print}

N.B.: When citing this work, cite the original article.

Original Publication:

Konrad J. Schönborn, Petter Bivall and Lena A. E. Tibell, Exploring relationships between students' interaction and learning with a haptic virtual biomolecular model, 2011, Computers and education, (57), 3, 2095-2105.

http://dx.doi.org/10.1016/j.compedu.2011.05.013

Copyright: Elsevier http://www.elsevier.com/

Postprint available at: Linköping University Electronic Press

http://urn.kb.se/resolve?urn=urn:nbn:se:liu:diva-68996 


\title{
Exploring relationships between students' interaction and learning with a haptic virtual biomolecular model
}

\begin{abstract}
This study explores tertiary students' interaction with a haptic virtual model representing the specific binding of two biomolecules, a core concept in molecular life science education. Twenty students assigned to a haptics (experimental) or no-haptics (control) condition performed a "docking" task where users sought the most favourable position between a ligand and protein molecule, while students' interactions with the model were logged. Improvement in students' understanding of biomolecular binding was previously measured by comparing written responses to a target conceptual question before and after interaction with the model. A log-profiling tool visualized students' movement of the ligand molecule during the docking task. Multivariate parallel coordinate analyses explored any relationships in the entire student data set. The haptics group produced a tighter constellation of collected final docked ligand positions in comparison with no-haptics students, coupled to docking profiles that depicted a more fine-tuned ligand traversal. Students in the no-haptics condition employed double the amount of interactive behaviours concerned with switching between different visual chemical representations offered by the model. In the no-haptics group, this visually intense processing was synonymous with erroneously 'fitting' the ligand closer distances to the protein surface. Students who showed higher learning gains tended to engage fewer visual representational switches, and were from the haptics group, while students with a higher spatial ability also engaged fewer visual representational switches, irrespective of assigned condition. From an information-processing standpoint, visual and haptic coordination may offload the visual pathway by placing less strain on visual working memory. From an embodied cognition perspective, visual and tactile sensorimotor interactions in the macroworld may provide access to constructing knowledge about submicroscopic phenomena. The results have cognitive and practical implications for the use of multimodal virtual reality technologies in educational contexts.
\end{abstract}

Keywords: interactive learning environments; multimedia systems; pedagogical issues; postsecondary education; virtual reality

\section{Introduction}

Current computing technology provides humans with the opportunity to experience and interact with virtual worlds designed to depict natural phenomena. Other than perceiving information visually and verbally, modern virtual environments engage a user's tactile sensory pathway. Haptics describes the perception of touch and force stimuli such as the texture, hardness and shape of objects (Lederman \& Klatzky, 1987).

Exploitation of haptics in immersive virtual reality models holds exciting directions for education and training (e.g. Richard, Tijou, Richard, \& Ferrier, 2006). Although studies investigating the role of haptics in science education are on the rise, Minogue and Jones (2006) have reported that little is known about the cognitive advantages of interacting with haptic virtual learning environments. In molecular life science education, where there is a growing emergence of such platforms in pedagogical contexts (e.g. Martin, Eid, \& El Saddik, 2008), there remains a serious lack of empirical inquiry on aspects of students' processing and learning with haptic virtual models. As part of addressing these shortfalls, we are investigating students' interaction and learning with a biomolecular haptic virtual model developed by Author2 et al. (2007), with a complementary objective of further contributing to empirically-based accounts of the cognitive merits and shortfalls of virtual reality environments for broader education contexts. 


\section{Literature review}

\subsection{Students' interaction and learning with haptic virtual models in science education}

Virtual reality models that incorporate visual and haptic experiences show great promise for science education because they can stimulate knowledge-building experiences and provide novel learning settings (e.g. Richard et al., 2006). Reiner (1999) has considered students' construction of force concepts during interaction with a virtual environment, while Dede, Salzman, Loftin, and Ash (2000) used a haptic virtual model to improve students understanding of kinematics. More recently, Wiebe, Minogue, Jones, Cowley, and Krebs (2009) investigated students' understanding of lever principles using a visuohaptic simulation.

In biology, Jones, Minogue, Tretter, Negishi, and Taylor (2006) explored the use of haptic virtual models in students' interpretation of viruses. In a further study on learning with a 3D haptic virtual model of an animal cell, Minogue, Jones, Broadwell, and Oppewall (2006) found that haptic experiences improved students' ability to navigate and interpret the cell environment. However, the study revealed no significant differences in cognitive benefit between students who received haptics and visual information versus those who only received visual feedback.

In a biomolecular context, Brooks, Ouh-Young, Batter, and Kilpatrick (1990) evaluated the effect of haptic feedback on expert biochemists' engagement with a virtual model that simulated interaction between two biomolecules. In two separate conditions, participants were required to "dock" a molecular drug candidate into the binding site of a protein molecule with force feedback enabled or disabled. Findings showed that participants who received haptic and visual feedback docked the ligand faster and that haptic feedback improved users' understanding of molecular force fields. In support of this result, Bayazit, Song, and Amato (2001) suggested that haptic feedback promotes users' understanding of biomolecular interactions because the molecular forces, which are already extremely complex to visualize, can be felt directly.

\subsection{Generating logging data to explore students' interaction with computer environments}

Data logging refers to the process of obtaining information about how a user interacts with a computer-based system. Technological research employs data logging to reveal users' exploratory preferences (e.g. Burigat, Chittaro, \& Ieronutti, 2008). However, data logging has been far less extensively applied to educational contexts where tracking events could provide fine-grained observations and detailed access into students' processes of interaction and learning (e.g. Huk, 2006: Kozma, 1994).

In a biology education context, Steinke, Huk, and Floto (2003) logged learners' paths through a computer environment concerned with cell biology phenomena. Logged data included the time on each viewed screen and the number of control activations (e.g. clicks of screen buttons). Students' paths through the system were tracked to map any relationship(s) between usage intensity and spatial ability. In a study on BioLogica, a hypermodel that aims to assist the learning of genetics, Buckley et al. (2004) analysed log files generated from students' interaction with the application to characterise students' problem-solving strategies. Tsui and Treagust (2007) confirmed the power of obtaining such data in BioLogica by showing that log files can uncover details about learners' interactions with computer models.

\subsection{Cognitive theories of students' interaction and learning with multimodal models}

Moreno and Mayer's (2007) cognitive-affective theory of learning with media (CATLM) considers learners' interaction and learning with multimodal environments through at least four tenets. Firstly, learners process different external modes through separate processing modalities. Secondly, each modality has a limited processing capacity within 
working memory. Thirdly, meaningful learning occurs when newly processed information is appropriately selected, organised and integrated with already existing knowledge. Fourthly, learners' cognitive engagement with the multimodal environment is mediated by motivational factors. Application of CATLM to learning with a multimodal system suggests that cognitive overload can occur when the processing demands of interacting with the environment are greater than the capacity of the learner's cognitive processing architecture (e.g. Wong et al., 2009). For example, Mayer and Moreno (2002) have shown that when strain is placed on visual working memory, fewer cognitive resources are available for building connections between the visual system and information from other channels (cf. Austin, 2009). Thus, in the latter case, learners could potentially learn more deeply when they are not required to process 'extra' pictorial information in visual working memory.

In contrast with CATLM, embodied learning is a theoretical perspective that considers bodily experiences as a key component of cognition. Here, human construction of knowledge is considered closely linked to sensorimotor interactions in the world (Wilson, 2002). In this regard, tactile sensations could stimulate learners to access and integrate embodied knowledge into their cognitive processing of abstract scientific concepts (e.g. Reiner, 1999). Therefore, experiencing a coordinated visual and tactile representation of biomolecular binding could have a potentially deep-seated influence on students' construction of knowledge concerning submicroscopic phenomena.

\section{Research aim}

The purpose of the study was to explore any relationships between students' processes of interaction with a virtual visuohaptic model of biomolecular binding and learning.

\section{Methodology}

\subsection{A haptic virtual model for representing the specific binding of biomolecules}

\subsubsection{The fundamental process of specific biomolecular binding}

The specific binding of one molecule to another is central to many biochemical reactions. A description of biomolecular binding is necessary to provide readers with the biological basis behind the haptic virtual system of interest to this study context. In this paper, biomolecular binding refers to a specific positioning and orientation of a ligand molecule (small molecule) into the binding site of a protein (large molecule) (e.g. Fig. 1A). The binding site is that location where the binding energy associated with attractive and repulsive forces that exist between the two molecules is at a minimum. Understanding binding is fundamental to learning molecular life science, and requires an appreciation of the dynamics of biomolecular interactions. When simulated by computer technology, the process of searching for this favourable position for the ligand is termed "docking". This term is a fundamental premise to the human-computer interaction dealt with in the current paper. In this regard, this paper concerns users' interaction with a haptic virtual model developed by Author2 et al. (2007).

\subsubsection{Technological aspects of the haptic virtual model}

The model allows a user to perform a docking, thereby simulating the process of biomolecular binding. It incorporates 3D stereo graphics and haptics in its representation of biomolecular binding (Fig. 1A). The system supports Phantom devices from Sensable Technologies, including the Phantom Desktop and Phantom Omni interfaces (Fig. 1B). 

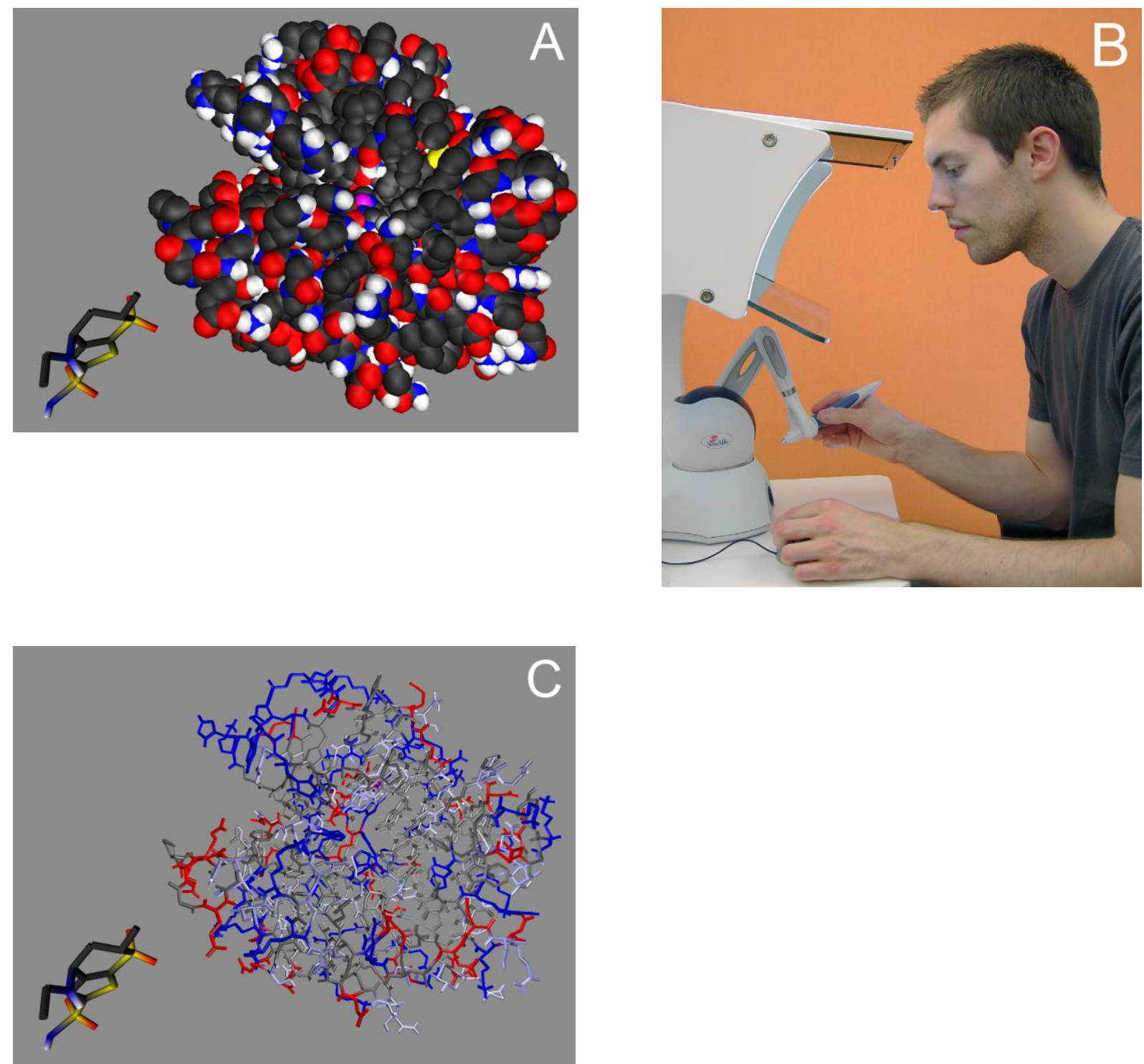

Fig. 1. A: Screenshot displaying the ligand (left) and protein (right) structures involved in the biomolecular binding. B: Photograph of a student using the visual and haptic hardware that renders the virtual model. C: Screenshot example of a different visual representation convention for depicting the protein structure.

Similar to most molecular visualization programs, the model can display any protein structure. The force acting on the ligand delivered through the haptic device as force feedback (Fig. 1B) is calculated in real-time based on computed force fields between the protein and the molecular structure of the ligand.

A haptic device is required to experience force feedback, but no such device is required to engage basic functions of the model. The model can be run with a standard computer and mouse for control of the ligand, albeit hardware with stereo graphics and a 3D navigation device is preferable (Fig. 1B). With respect to examining student data in the present paper, the model was used with semi-immersive workbenches that provided stereo graphics, and with the virtual ligand attached to the tip of the haptic device. A (3D) space 
mouse enabled individual control of the position and rotation of the protein (Fig. 1). The model can be run both with and without haptic feedback enabled, where in the latter case, the haptic device functions as a 3D navigation tool for controlling the position of the ligand.

\subsubsection{Interactive aspects of the haptic virtual model}

The user manoeuvres a pen-like stylus to manipulate the ligand molecule and a space mouse to control the protein, while simultaneous attractive and repulsive forces are generated as the ligand is moved close to the binding site of the protein. In this paper, students' movement of the haptic device with their hand (Fig. 1B), which corresponds to the movement of the virtual ligand viewed by the user (Fig. 1A), will be referred to as traversal of the ligand. Such ligand traversal is an important component of considering users' humancomputer interaction in the current research context.

Display of the protein molecule can be selected from a variety of visual modes that are typically used in molecular software, and which are considered conventional visual representations encountered in molecular life science education. A possible sixteen different visual representational modes (e.g. Fig. 1A and C) can be engaged at any one time by pressing the appropriate keys on a keyboard. In this paper, any behaviour concerned with engaging the different visual modes on offer will be referred to as representational switching. Therefore, a single representational switch consists of a student's activation of one of the available sixteen visual representation modes. We hypothesise that students' representational switching is intimately coupled to visual cognitive processing and we consider it a crucial feature of human-computer interaction in the present research context.

Apart from using the haptic device to move the ligand, the user can adjust the angle of attachment of the haptic device to the ligand. Since the haptic hardware does not allow the pen-like stylus (Fig. 1B) (and therefore the ligand) to rotate through $360^{\circ}$, such a "grip change" is required if the user wishes to orientate the ligand in a specifically desired position. In this paper, we refer to initiating a grip change as that behaviour concerned with pressing the grip change button on the stylus, establishing a desired angle of attachment of the ligand, and then releasing the button. We consider such behaviours as a crucial component of humancomputer interaction in the present study.

\section{2. $\quad$ Student participants and teaching context}

The present paper is concerned specifically with reporting the interactive part of the data garnered from a broader set of studies (e.g. see Author2 et al., in press) collected from 20 postgraduate life science students enrolled in a course titled Biomolecular Interactions. The course is part of the Masters program in Chemical Biology at Linköping University, Sweden. The central educational objective of the course is to provide students with a conceptual understanding of the process of biomolecular binding. The 20 course participants ( 8 females and 12 males) were assigned to one of two conditions, one "experimental" and one "control" group. The experimental (haptics) group $(\mathrm{n}=10)$ interacted with the model with force feedback activated while the control (no-haptics) group $(n=10)$ received no force feedback. All students participated voluntarily in the study and all had a similar educational background. As stated in Author2 et al. (in press), students' assignment to the experimental or control condition was random except that the two groups were designed so that gender and prior knowledge were similarly distributed. Since the current paper focuses on describing and exploring the relationships between students' interaction with the model and previously observed conceptual gain, please consult Author 2 et al. (in press) for specific details regarding the between-groups pre- and posttest experimental design pertaining to the data collection. 


\subsection{Collection of data}

\subsubsection{Students' responses to a target question before and after exposure to the model}

Before and after exposure to the model, all students responded to a written conceptual question that required describing the process of a ligand binding to a protein. Responses to this pre-/posttest item (PPI) were the measure of any changes in students' conceptual understanding of biomolecular binding after interacting with the model. The design behind the PPI was that students' comprehension of biomolecular binding would require a demonstration of a range of biomolecular concepts of considerable scientific depth.

\subsubsection{Administering of a spatial ability test before exposure to the model}

Before interaction with the model, all students completed a pencil-and-paper 2D rotation spatial ability test developed by Ekstrom, French, and Harman (1976). Administering the spatial test was based on the premise that spatial aptitude is an important component of visual literacy in molecular life science. Hence, we had an interest in exploring any relationships between students' spatial aptitude and interaction and learning with the model.

\subsubsection{Logging students' interaction with the model during performing of a docking task}

Following the pretest and spatial ability test, students in each haptics (experimental group) or no-haptics (control group) condition interacted with the model to perform a docking task that required each student to find the best binding position between a ligand and protein (Fig. 1). Students' interaction with the model during the docking task was tracked in log files, which are the human-computer interactive data upon which the present study finds its basis. Here, the log files stored the coordinates of the traversal of the ligand molecule (relative to the protein molecule) in virtual 3D space at two-second intervals. The force value obtained from the potential field at each ligand position (also logged in the no-haptics group where force feedback was deactivated) was also logged. Interactive time was defined as the sum of those $\log$ entries where any force was obtained, since these entries corresponded to periods where we were certain students were actively interacting with the model. Other logged data included the two earlier defined interactive behaviours, namely representational switches and grip changes, as well as the point in the task where students "saved" their docking. Here, the accuracy of each docking (the final saved position of the ligand) was measured in terms of the Root-Mean-Square (RMS) value. The RMS value compared a student's saved docked ligand position with the "correct" docking. Low RMS values inferred better dockings and RMS values were used as the measure of docking accuracy.

\subsection{Data Analysis}

As stated, log files were the data for analysing students' interaction with the model. However, exploring relationships between interaction and learning with the model required contrasting prior analyses of students' responses to the PPI recently reported in Author 2 et al. (in press).

\subsubsection{Analysis of students' responses to the PPI}

Since the focus of the presently reported study is on exploring any relationships behind the significant gain in learning with the model previously observed by Author2 et al. (in press), please consult the latter article for details regarding the statistical treatment of students' responses to the PPI. A 'full' score on the PPI required students to reflect a deep understanding of various aspects of biomolecular binding. 


\subsubsection{Treatment of students' spatial ability scores}

Relationships between students' spatial ability scores and students' representational switching and grip changing activity with the model were considered. In addition, relationships between spatial ability and learning gain scores from the PPI were also analysed.

\subsubsection{Analysis of students' interaction with the model during the docking task}

Regarding students' traversal of the ligand, logged data obtained from the docking task was visualized as a profile pattern within a 3D Cartesian coordinate system. Each logged position of the ligand was depicted as a sphere. Larger spheres corresponded to positions of greater force magnitude (also recorded in the no-haptics group), while elapsed time-on-task was imparted through a black-to-white contrast gradient (see Fig. 4).

ViewerLite (Accelrys, 2002) was applied to visually analyse students' final saved docked positions. The collection of dockings in each haptics and no-haptics condition, as well as students' final dockings relative to the correct ligand position were analysed (see Fig. 2). This analysis provided a qualitative appreciation of the otherwise quantitative RMS values.

\subsubsection{Multivariate parallel coordinate analysis of the entire student data set}

A parallel coordinate (PC) system was used to perform a multivariate analysis of relationships between variables across the entire data set. ${ }^{1} \mathrm{~A}$ PC plot consists of a set of parallel vertical axes, one axis for each variable of interest (e.g. Johansson, 2008). Data across five variables (i.e. five parallel axes) were plotted, namely Learning gain on PPI (\%), Grip changes, Spatial ability score (\%), Representational switches, and Mean force experienced by the ligand $(u / s e c$.$) . We considered these variables essential to exploring any links between$ human-computer interaction and learning in the current research domain. A PC plot represents data by a set of polylines that intersects each of the parallel axes at those points corresponding to different values in the data (see Fig. 3). Each polyline corresponds to the data for one student. Please access the video clip in footnote 1 for a narrated explanation of the PC system and associated analysis.

Data analysis exploited three specific analytical features by using a mouse. One feature involved filtering the number of polylines (i.e. number of students) that were displayed on the plot at any one time. A further procedure allowed any of the five parallel axes to be dragged left or right over another so that they appeared in any order of preference. Lastly, clicking on any of the polylines rendered the clicked polyline with a thicker black line, which allowed the datum for an individual student to be visualized across all five axes.

\subsection{Overall data analysis framework}

Data analysis was interpretive and in line with other empirical investigations on pedagogical innovations (e.g. Tsui \& Treagust, 2007). Data analysis aimed to explore students' interaction with the model within the defined educational context, and describe any patterns of observation bound to this situated context. The direction of data analysis proceeded from first elucidating any overall differences between the two conditions, then analysing data across and within each group, towards finally exploring individual student cases. Data was analysed at two levels. Cross-case analyses explored any relationships across students' interaction with the model in the haptics and no-haptics conditions. Within-case analyses explored each student's interaction with the model (e.g. Wu, Krajcik, and Soloway, 2001). Although described linearly, this process often encompassed multidirectional analyses between cross-group, cross-case and within-case data (e.g. Schönborn \& Anderson, 2009).

\footnotetext{
${ }^{1}$ A narrated video clip demonstrating how PC analysis was performed in the present study can be accessed directly at http://www.ep.liu.se/videos/10.1016j.compedu.2011.05.013/PC_video.avi.
} 


\section{Results}

Findings are structured in three sections. Each successive section supplies a further level of detail consistent with the analytical direction of presenting overall relationships towards individual cases. Firstly, section 5.1. provides an overall comparison of students' data from the haptics and no-haptics conditions. Secondly, section 5.2. reports the results from the PC analysis of relationships across five variables related to students' interaction and learning with the model. Thirdly, section 5.3. presents examples of individual log file profiles obtained from the docking task.

5.1. Overall cross-group comparison of haptics and no-haptics conditions on students' interaction and learning with the model

An overall quantitative comparison of the haptics ${ }^{2}$ and no-haptics conditions on students' interaction and learning with the model is presented in Table 1.

Table 1. Overall descriptive statistics for a group comparison of the haptics and no-haptics conditions in the study.

\begin{tabular}{|c|c|c|c|c|c|c|c|c|}
\hline \multirow[t]{2}{*}{ Measure } & \multicolumn{4}{|c|}{ Haptics group } & \multicolumn{4}{|c|}{ No-haptics group } \\
\hline & Mean & Median & Max. & Min. & Mean & Median & Max. & Min. \\
\hline \multicolumn{9}{|l|}{$\begin{array}{l}\text { Obtained from written } \\
\text { responses: }\end{array}$} \\
\hline Learning gain on PPI (\%) & 15.0 & 15.0 & 40.0 & 0.0 & 3.5 & 0.0 & 20.0 & -10.0 \\
\hline Spatial ability score (\%) & 69.7 & 66.3 & 100.0 & 41.3 & 68.2 & 68.8 & 86.3 & 47.5 \\
\hline \multicolumn{9}{|l|}{$\begin{array}{l}\text { Obtained from } \\
\text { log files: }\end{array}$} \\
\hline Docking accuracy (RMS) & 4.9 & 3.9 & 7.5 & 3.2 & 5.2 & 5.0 & 10.1 & 2.8 \\
\hline Interactive time (s) & 268.0 & 238 & 532 & 72 & 341.8 & 296 & 744 & 72 \\
\hline Travelled distance $(\AA)$ & 168.8 & 113.9 & 486.4 & 41.9 & 87.8 & 97.3 & 159.6 & 18.4 \\
\hline $\begin{array}{l}\text { Force feedback rate } \\
\text { (u/sec.) }\end{array}$ & 2263.5 & 1487.2 & 6136.3 & 173.3 & 13653.1 & 15027.8 & 20175.6 & 6484.8 \\
\hline $\begin{array}{l}\text { Representational } \\
\text { switches }\end{array}$ & 6.1 & 4 & 23 & 0 & 13.9 & 7.5 & 40 & 0 \\
\hline $\begin{array}{l}\text { Grip initialisation } \\
\text { changes }\end{array}$ & 10.9 & 8 & 28 & 2 & 17.3 & 15.0 & 30 & 5 \\
\hline
\end{tabular}

Table 1 contains two overall types of data namely, data obtained from students' written responses and data obtained from the log files.

\footnotetext{
${ }^{2}$ Since one student in the haptics group did not save his/her final docked position, all values except for learning gain were calculated for nine (of the ten) students assigned to the haptics condition.
} 


\subsubsection{Comparison of written student data obtained from the PPI and spatial ability test}

Students' responses to the PPI before and after interaction with the model yielded low mean scores that ranged from 3.5 to 5.4 (out of a maximum of 10) across both conditions. This confirmed the assumption that providing a conceptual answer to the PPI was a challenge even for the Masters-level students who participated in the study. No real difference in both groups' mean and median spatial ability values were observed prior to interaction with the model (Table 1). Nevertheless, as earlier reported by Author 2 et al. (in press), a statistically significant learning gain of $15 \%$ was obtained in the haptics group in comparison with an average gain of $3.5 \%$ in the no-haptics group (Table 1), after interacting with the model. Respective median gain scores of $15 \%$ versus $0 \%$ also describe this difference (Table 1).

\subsubsection{Comparison of data obtained from students' interaction with the model}

\subsubsection{Interactive time, docking accuracy, and movement of the ligand molecule}

The haptics group spent less interactive time with the model on average (268 sec. versus 342 sec., Table 1). However, there was hardly any difference in docking accuracy between both groups (mean RMS of 4.9 versus 5.2), although the largest range between the least and most accurate docking occurred in the no-haptics condition. Upon interacting with the model to perform the docking task (cf. Fig. 1), the haptics group moved (traversed) the ligand molecule an average distance of $168.8 \AA$, relative to the protein molecule, in comparison with an average traversal of $87.8 \AA$ in the no-haptics group (Table 1). Nevertheless, this difference is less evident when the median values for each of the haptics (113.9 $\AA$ ) and no-haptics $(97.3 \AA$ ) conditions are considered.

Although there was hardly any quantitative difference in mean docking accuracy between the groups (as measured by the RMS value), it is demonstrated qualitatively in Fig. 2A that the haptics group (red) produced a less dispersed and 'tighter' constellation of final docked ligand positions than the no-haptics group (blue), relative to the position of the "correct" docking (Fig. 2B). This observation is probably consistent with the greater range in RMS values (docking accuracies) obtained in the no-haptics condition (Table 1).

\subsubsection{Force feedback, representational switches, and grip changes}

Had the no-haptics group received force feedback during the task (and assuming that identical dockings as in Fig. 2A were obtained), it would have been delivered at a much greater average rate than the haptics group experienced $(13653 \mathrm{u} / \mathrm{sec}$ versus $2263 \mathrm{u} / \mathrm{sec}$, Table 1). Stated differently, students in the no-haptics group positioned the ligand molecule closer distances to the surface of the protein over time than did the haptics group. This marked interactive behaviour has important implications for reflecting on the way students used the no-haptics version of the virtual technology as a problem-solving tool.

On average, during the docking task, the no-haptics group made more than double the number of visual representational switches (mean 13.9, median 7.5) between different visual chemical conventions (e.g. Fig. 1A and C) in comparison with the haptics group (mean 6.1, median 4). A similar scenario is evident in the average number of grip changes engaged by each group when positioning and orientating the ligand during docking, respectively (17.3 versus 10.9). Differences in grip change frequency are reinforced by comparing the median values obtained in the no-haptics (15) and haptics (8) condition. Such differences in these human-computer interactions between the conditions have consequences for interpreting the underlying cognition related to users' engagement with virtual systems during learning. 

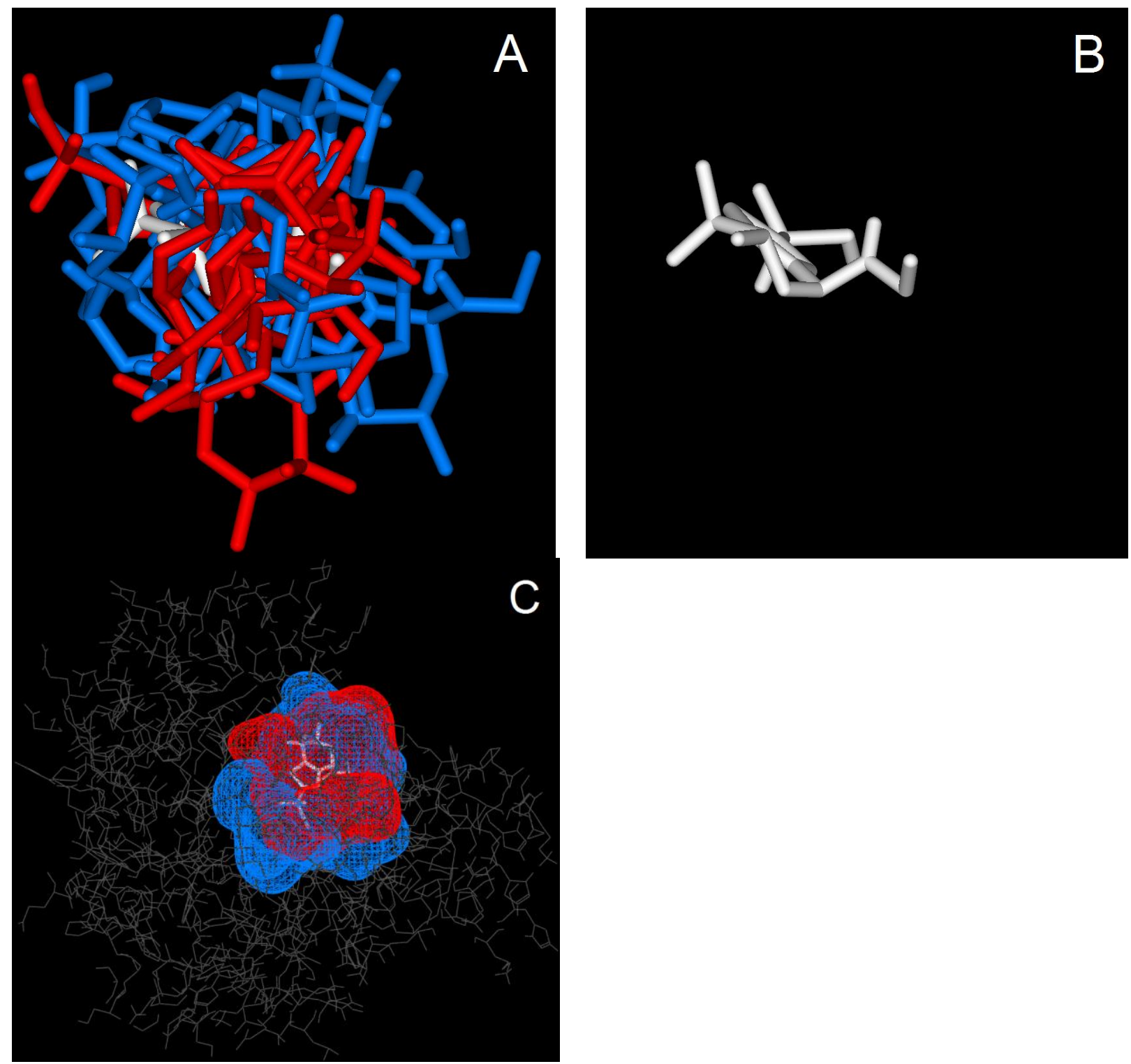

Fig. 2. A: Collection of final positions of each student's saved docked ligand in the haptics (red) and no-haptics (blue) conditions, shown from a top view that looks down onto the binding site (the protein is not displayed). B: Correct docking position of the ligand (white). C: Overall molecular volume occupied by each group of students' collected final saved docked ligand positions in the haptics (red) and no-haptics (blue) conditions. The relative position of the protein structure is depicted in grey, and the correct ligand docking solution is displayed in white. Generated with ViewerLite from Accelrys Software Inc.

\subsection{Relationships between students' interaction with the model and learning obtained from parallel coordinate analyses}

This section presents results from the PC analysis that explored relationships across the entire student data set. Please consult the link to the narrated video clip in footnote 1 when reading this section. 


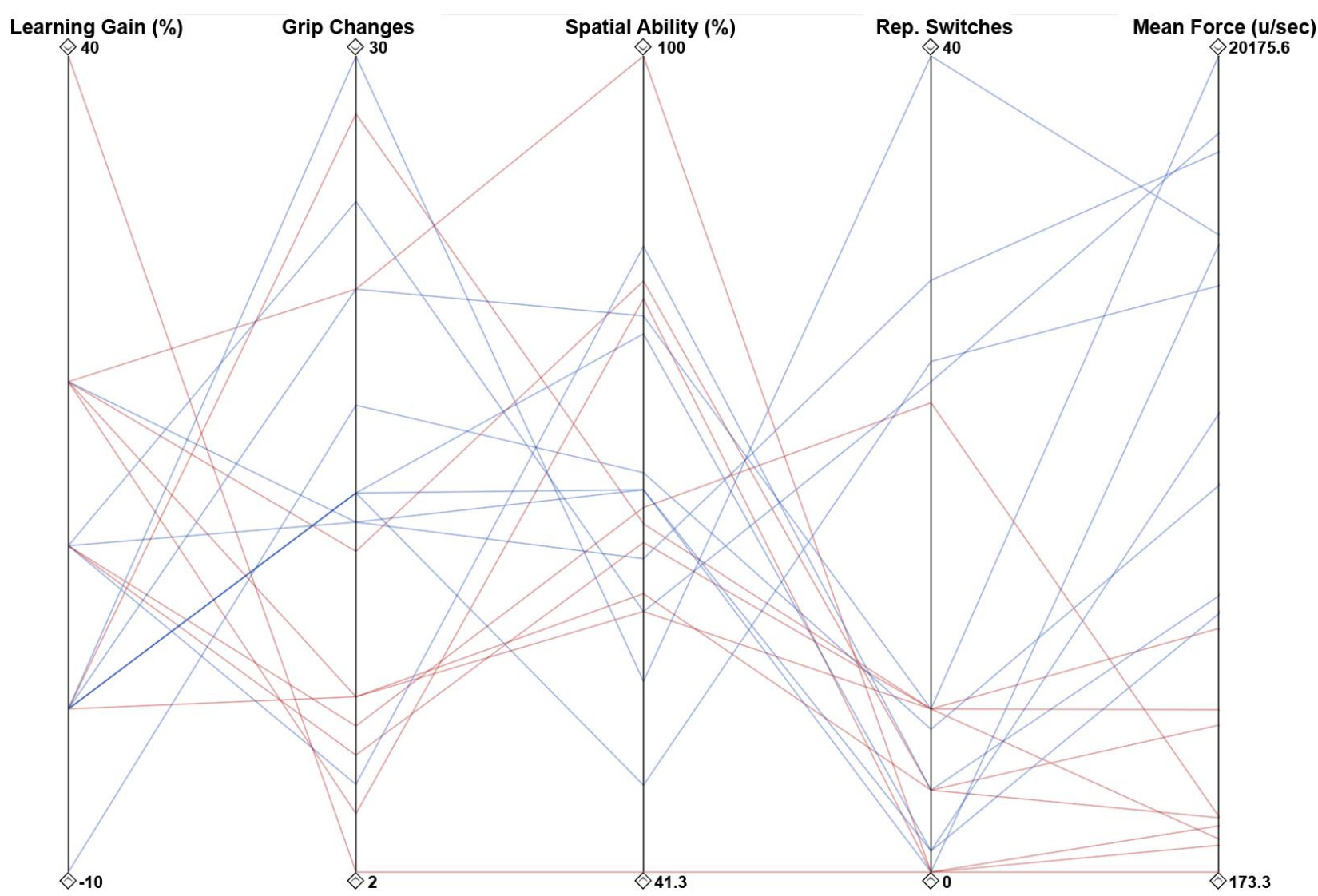

Fig. 3. Multivariate parallel coordinate (PC) plot displaying the entire data set obtained in the study from all students assigned to the haptics (red polylines) and no-haptics (blue polylines) conditions across five variables, which are labelled at the top of each parallel axis. Interpretation of the data in the plot is complemented by viewing the narrated video (http://www.ep.liu.se/videos/10.1016j.compedu.2011.05.013/PC_video.avi).

Fig. 3 represents the entire data set obtained from all individual participants in the study assigned to the haptics (red polylines) and no-haptics (blue polylines) conditions across five variables (parallel axes), namely, Learning gain on PPI (\%), Grip initialisation changes, Spatial ability score (\%), Representational switches, and Mean force experienced by ligand (u/sec.). We considered these variables central to exploring any relationships between interaction and learning with the virtual reality technology of interest to the present computers and education research context.

\subsubsection{Relationships between learning gain, representational switches and grip changes}

Fig. 3 (and the narrated video) reveals the overall pattern that higher learning gain scores concerning a conceptual understanding of biomolecular binding were associated with fewer grip changes. Furthermore, fewer grip changes tended to be associated with students in the haptics (red lines) condition, while larger numbers of grip changes tended to be associated with students in the no-haptics group. Accompanying this trend was the overall positive correlation between engaging grip changes and performing representational switches during the docking task. Students who engaged high numbers of representational switches tended to be from the no-haptics group (Fig. 3A). In fact, the four students who showed the highest visual representational switching activity in the study were all from the no-haptics condition. At the same time, students who showed higher learning gains tended to engage fewer representational switches, and were from the haptics group (Fig. 3A). For instance, the student who demonstrated the highest learning gain in the study (red polyline at the top of the 
learning gain axis in Fig. 3) did not make a single representational switch and performed only two grip changes (Fig. 3). Such trends could provide a window into the relationship between users' interaction with the virtual environment and their construction of core science concepts.

\subsubsection{Relationships between mean force experienced by the ligand, representational switches and grip changes}

As per Fig. 3 (and the narrated video), all the blue polylines appear above the red polylines on the Mean Force axis. From a group perspective, this demonstrates that students in the no-haptics group brought the ligand closer distances to the protein surface than did the haptics group while interacting with the model. From an educational standpoint, positioning of the ligand in such close proximities to the protein surface would be physically impossible in the corresponding real submicroscopic scenario. Interestingly, in the no-haptics condition, such positioning of the ligand closer and deeper 'into' the protein surface (cf. Fig. 2C) was associated with more representational switches and grip changes. This result reveals a clear connection between students' interpretation of visual features of the system and complementary patterns of human-computer interactive behaviours.

\subsubsection{Relationships between spatial ability, representational switches and learning gain}

From Fig. 3 (and the narrated video), there is a general pattern indicating that students with a spatial ability score of greater than $80 \%$ engaged fewer representational switches. A case in point is that the student who scored maximum on the spatial ability test did not make a single representational switch. The same relationship is also evident in the no-haptics group in that students with lower spatial ability scores tended to make frequent representational switches (Fig. 3). Lastly, despite the fact that there was no association between spatial ability and learning gain in the study at the group level, the four students who displayed learning gains in the no-haptics group, all displayed a spatial ability of greater than $60 \%$ (Fig. 3). These trends could offer insight into what extent particular interactive behaviours with computing technology are influenced by users' existing cognitive and conceptual aptitudes.

\subsection{Docking profiles of individual students' interaction with the model}

In completion of presenting findings in the direction of overall relationships to specific details pertaining to individual cases in the current computers and education research context, this section presents examples of individual log profiles of how students traversed the ligand during the docking task.

Fig. 4A and B are representative of profiles yielded from two students who performed the docking task with haptic feedback enabled and disabled, respectively.

\subsubsection{Docking profiles generated from individual students in the no-haptics condition}

Visualized docking profiles obtained from the no-haptics condition revealed dispersed collections of ligand positions. For example, as profiled in Fig. 4B, the student's movement of the ligand towards the protein appears indiscriminate with respect to any feasible binding area. The larger spheres rendered at later periods during the docking task suggests that final dockings occurred when the ligand was in close spatial proximity to the surface of the protein surface (cf. Fig. 2C). As demonstrated earlier, such positioning of the ligand was coupled to an increased visual representational switching activity. In the case of the student depicted in Fig. 4B, this high visual processing behaviour was associated with a zero learning gain. 


\subsubsection{Docking profiles generated from individual students in the haptics condition}

As represented in Fig. 4A, profiles obtained from students who received force feedback showed a more defined quality to their movement of the ligand. For example, Fig. 4A depicts a channel-like traversal of the ligand, indicative of a localised docking exploration

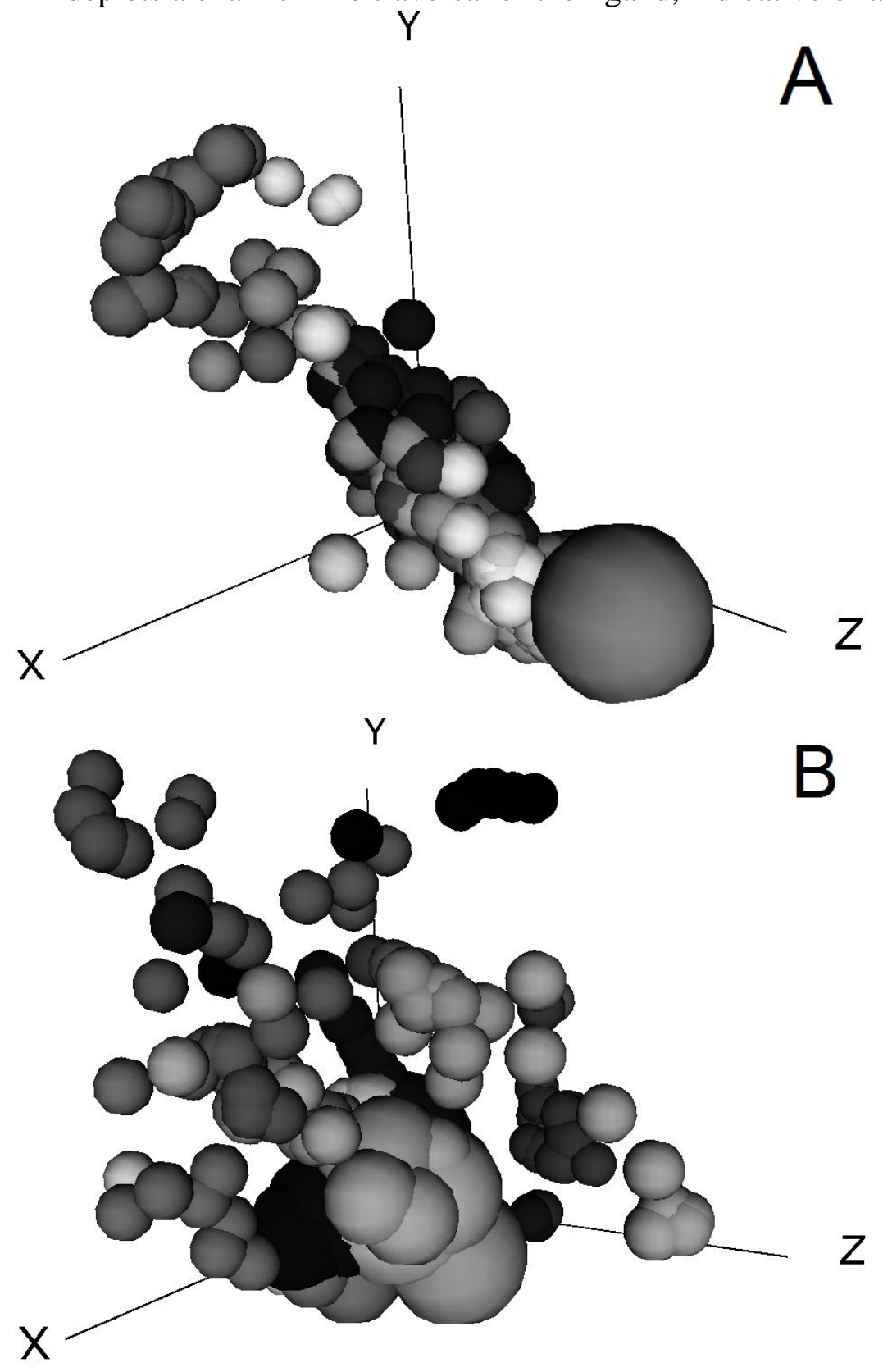

Fig. 4. Two examples of docking profiles showing how individual students traversed the ligand relative to the protein over time in a $3 \mathrm{D}$ Cartesian coordinate $(\mathrm{X}, \mathrm{Y}$, and $\mathrm{Z}$ ) system. A: A profile obtained from the haptics condition. B: A profile obtained from the no-haptics condition. In both profiles, darker shaded spheres represent earlier periods in the docking task, while more lightly shaded spheres indicate later times. Relatively larger sized spheres depict positions of higher force values.

(cf. Fig. 2A). When elapsed time-on-task is traced via the dark-to-light contrast gradient, it becomes apparent that the student profiled in Fig. 4A moved the ligand back-and-forth multiple times within the trajectory volume while seeking a feasible docking position. Also, in comparison with Fig. 4B, it is evident that distinctly lower force values were associated with 
docking the ligand in the haptics condition as confirmed by the smaller ratio of large-sized spheres in the location of what was interpreted as the binding site. The profile in Fig. 4A is from the student who demonstrated the highest learning gain in the study, and who did not deploy a single representational switch during the entire docking task.

\section{Discussion}

\subsection{The role of force feedback in students' execution of the docking task}

In contrast with the no-haptics condition (e.g. Fig. 4B), haptic feedback could offer a visuotactile 'map' that allows for a physically realistic movement of the ligand (e.g. Fig. 4A). The tighter constellation of students' final docked ligand positions in the haptics group (Fig. 2A) supports the channel-like docking traversal in Fig. 4A. Although such a 'focusing' effect may not necessarily result in a more accurate docking (Table 1), it certainly helps define potentially feasible binding-areas on the protein surface. Such possible benefits of the haptic virtual technology in the current context could be extended to other training domains (e.g. simulated surgery) where such focused interaction might be practically favourable.

The traversal paths obtained in the haptics condition mirror the project GROPE findings (Brooks et al., 1990) in that users proceeded more 'directly' to the binding site. Although project GROPE revealed that ligand trajectory path-lengths were shorter in the presence of force feedback, our study showed that haptics students traversed the ligand further, on average. This difference could be explained by the notion that due to the novel multimodal experience, students' in our study may have been motivated to "try the model out" more. Evidence for this hypothesis lies in those haptic cases where the ligand was traversed back-and-forth multiple times during the docking task (e.g. Fig. 4A). Overall, this result suggests that users' engagement with virtual multimedia systems could be a motivating factor in itself, which could be exploited for pedagogical purposes.

Our finding that users in the haptics condition spent less interactive time with the model in completion of the docking task also confirms results from project GROPE (Brooks et al., 1990). However, a study by Wiebe et al. (2009) revealed that students in a visual and haptic group spent a significantly longer time interacting with virtual haptic levers. It would be noteworthy to study relationships between time on task and students' interactive behaviours in different educational contexts that use visuohaptic technologies for learning.

\subsection{Students' interaction with the model in light of the nature of biomolecular binding}

Since students in the haptics condition positioned their ligands at higher altitudes above the binding-site overall than the no-haptics group (Fig. 2C), one clear pedagogical advantage of haptic feedback is that it provides an explicit perceptual experience that intermolecular forces are a major determinant in biomolecular binding. In terms of the prominent differences in force-related values across the two conditions, Fig. $2 \mathrm{C}$ provides qualitative evidence that overall, the no-haptics group tended to depress the ligand 'deeper' into what was interpreted as the binding 'cavity' on the protein, corresponding to a realistically unfavourable binding position. Coupled to this behaviour was the strikingly high visual representational switching activity in the no-haptics group.

Readers may argue that the link between haptics and a gain in learning is not surprising in this educational context. We contend that it is not merely the perception of haptics that induces benefit, but rather the realistic awareness that it imparts (cf. Brooks et al., 1990). By the same reasoning, our findings could have implications for reflecting upon other pedagogical environments that intend exploiting visuohaptic tools for learning in general. 


\subsection{Cognitive processes related to interaction with visuohaptic models in learning}

The extensive number of representational switches observed in the no-haptics group as a whole suggests that these students relied heavily on viewing different chemical features of the protein to seek the 'best' binding position for the ligand. This activity would have involved an intense visual analysis of different molecular features (e.g. Fig. 1A and C), and required a continuous translation between visual representations (e.g. Ainsworth, 1999; Schönborn \& Bögeholz, 2009). Doing so would have meant holding multiple mental visual representations in working memory at once (Moreno \& Mayer, 2007), which could have placed strain on visual working memory. Furthermore, in the no-haptics condition, we propose that performing grip changes also involved a high degree of visual processing in determining the best visuospatial alignment of the ligand. At the same time, learners with low spatial aptitudes would have had to direct large processing efforts towards manipulating multiple mental visual representations at once (cf. Mayer \& Moreno, 2003). Hence, we contend that the perception of force feedback in the haptics group could have actually compensated for any low spatial abilities.

In contrast with the no-haptics group, students in the haptics group could build one-toone correspondences in the sharing of information between visual and tactile modes. This may have placed less demand on the visual pathway alone, and released valuable working memory for learning processes (e.g. Wong et al., 2009). In fact, there is recent evidence for the premise that visual information can be "offloaded" onto the haptic channel in high load situations (e.g. Enriquez \& MacLean, 2008). In the haptics group, such offloading of the visual channel could have increased cognitive capacity for linking new mental representations to already existing knowledge. The processing dimensions offered here could perhaps be extended to consider learning in other domains that employ visuohaptic computing systems for communicating abstract concepts.

\subsection{Contrasting the study with general aspects of multimodal information processing}

In 1997, Tindall-Ford, Chandler and Sweller showed that combined auditory and visual information resulted in superior learning in comparison with a visual-only format. An explanation of this modality effect is the idea that a coordination of perceptual modalities induces an "effective expansion of working memory", which allows the individual to direct processing power to "learning" (Tindall-Ford et al., 1997, p. 257). In a chemistry education context, Tasker and Dalton (2006) demonstrated that, "working memory can be expanded slightly by mixing the senses used to present information" (p. 148). Similarly, Kalyuga, Chandler and Sweller (2004) have stated that dual-mode audiovisual presentation, "effectively eliminates cognitive overload of the visual channel" (p. 568). Similarly, results of the present study could support the notion of a visuohaptic modality effect.

With respect to science education, other research has shown little (or no) learning gain with virtual models that combine visual and haptic information versus visual feedback alone. For example, Minogue et al. (2006) found only small differences in cognitive gain after students' interaction with a haptic model of a cell. Moreover, recent work by Wiebe et al. (2009) showed that learners' interaction with a visual-haptic simulation of levers did not lead to an increased understanding of lever principles. An extrapolation of our results to education contexts at large is that the learning benefit of haptics is most likely a function of the pedagogical context and nature of the intended learning task.

\subsection{Implications of cognitive theory for explaining learning with visuohaptic models}

Our study suggests that a 3D visual display coordinated with haptics offered by a model in the macroworld could provide an embodied experience for understanding an aperceptual submicroscopic process. Hence, one might speculate that the tactile sensation 
allows translation of "feeling of the force fields" into an understanding of the realistic biomolecular scenario.

This study makes no claim whatsoever on the perceptual limits of the haptic channel as a sensory modality on its own. The results only go as far as revealing what humancomputer interactions could be responsible for improved learning in this context, and that conceptual understanding of binding is compromised when visual working memory loads are high. However, Ainsworth (2008) suggests that what modality-specific theories often ignore is the relationship between limited capacity and the "constructivist nature of human cognition", in that other factors such as individual preferences, attitude and previous experience can all influence the construction of knowledge from multimodal platforms.

A final cognitive view is that human visual and haptic perception is intertwined. For instance, Sathian, Zangaladze, Hoffman and Grafton (1997) used positron emission tomography (PET) to show that a tactile discrimination task activated brain areas specialised for visuospatial processing. With respect to virtual visuohaptic learning environments, creating links between neuroscience and pedagogy is an urgently needed direction of research enquiry.

\subsection{Issues of validity in the study}

This paper had its genesis in exploring the human-computer interactive processes behind learning gains with the model observed by Author 2 et al. (in press). Given the limited sample size $(n=20)$, we acknowledge the threat to external validity in any generalisability of the findings. However, the focus of this study was on describing and exploring any differences in students' interactive patterns when using the model for learning in the defined educational context, rather than making generalisations to the larger population. Support for the content validity of the PPI as a learning measure is in the fact that scientific responses to the PPI were obtained in both conditions, as well as by a semantic analysis of students' responses reported elsewhere. One limitation of the log data-collection was that samples were logged at two-second intervals, which would have excluded interactive events between successive log entries. Lastly, it may be early to claim high construct validity in any relationship between the exposed interactive behaviours and the delivered learning outcomes. However, such is the nature of any explorative study in educational research.

\section{Conclusions}

The potential benefits of the reported visuohaptic model can be summarised from an interactive, cognitive and conceptual perspective, which all have possible wider implications for the use of other multimodal virtual reality systems in contemporary education and training contexts. Firstly, from an interactive viewpoint, the benefits of the virtual model with force feedback activated are that:

- Experiencing haptic feedback helps students to perform fine-tuned ligand traversals.

- Force feedback offers students a sensory opportunity for 'error correction'.

- Haptic feedback helps students to localise feasible docking positions more efficiently.

Secondly, from a cognitive standpoint, the specific benefits of deploying the visuohaptic model are that:

- Bimodal haptic and visual referential connections could offload the visual channel by decreasing visual working memory processing.

- Knowledge integration is promoted by simultaneously coordinating visuospatial and tactile perception.

- Processing 3D information in a visuohaptic form could compensate for students with low spatial aptitudes. 
Lastly, in terms of conceptual understanding, the benefits of the visuohaptic model are that:

- Macroscopic sensorimotor experiences can stimulate the construction of scientific knowledge about the submicroscopic world.

- Force feedback actively imparts the scientific construct that dynamic interactions between molecules play a major role in biomolecular binding.

In conclusion, this study has uncovered interactive relationships associated with an improvement in students' learning about biomolecular binding after exposure to a haptic virtual model. Although the exact perceptual role of the haptic modality in learning is still under investigation, this study could be applied to pedagogical contexts where virtual visuohaptic models are envisioned for the construction of abstract yet core scientific concepts. Future research will further explore the role of embodied cognition in translating macroscopic experiences into knowledge about submicroscopic phenomena.

\section{Acknowledgments}

The Swedish Research Council (VR Grant 2008:5077) and a postdoctoral scholarship from Linköping University supported this research. The authors acknowledge Mr. Joel Nises for programming the log file profiling tool, and are grateful to Dr. Gunnar Höst and Prof. BengtHarald Jonsson for valuable discussions.

\section{References}

Accelrys Software Inc. (2002). ViewerLite version 5.0. San Diego: Accelrys Software Inc. <http://www.accelrys.com>

Ainsworth, S. (1999). The functions of multiple representations. Computers \& Education, 33(2-3), 131-152.

Ainsworth, S. (2008). How should we evaluate multimedia learning environments? In J.-F. Rouet, R. Lowe, \& W. Schnotz, Understanding multimedia documents (pp. 249-265). New York: Springer.

Austin, K. A. (2009). Multimedia learning: Cognitive individual differences and display design techniques predict transfer learning with multimedia learning modules. Computers \& Education, 53(4), 1339-1354.

Author2 et al. (in press). Science Education.

Author2 et al. (2007).

Bayazit, O. B., Song, G., \& Amato, N. M. (2001). Ligand binding with OBPRM and user input. Paper presented at the IEEE international conference on robotics and automation, Seoul, Korea.

Brooks, F. P., Ouh-Young, M., Batter, J. J., \& Kilpatrick, P. J. (1990). Project GROPE Haptic displays for scientific visualization. ACM Computer Graphics, 24(4), 177-185.

Buckley, B. C., Gobert, J. D., Kindfield, A. C. H., Horwitz, P., Tinker, R. F., Gerlits, B., Wilensky, U., Dede, C., \& Willett, J. (2004). Model-based teaching and learning with BioLogica $^{\mathrm{TM}}$ : What do they learn? How do they learn? How do we know? Journal of Science Education and Technology, 13(1), 23-41.

Burigat, S., Chittaro, L., \& Ieronutti, L. (2008). Mobrex: Visualizing users' mobile browsing behaviors. IEEE Computer Graphics and Applications, 28(1), 24-32.

Dede, C., Salzman, M., Loftin, R. B., \& Ash, K. (2000). The design of immersive virtual learning environments: Fostering deep understandings of complex scientific knowledge. In M. Jacobson, \& R. B. Kozma, Innovations in science and mathematics education: Advanced designs for technologies of learning (pp. 361-414). Mahwah, NJ: Erlbaum. 
Ekstrom, R. B., French, J. W., \& Harman, H. H. (1976). Manual for kit of factor-referenced cognitive tests. Princeton: Educational Testing Service.

Enriquez, M., \& MacLean, K. (2008). The role of choice in longitudinal recall of meaningful tactile signals. Paper presented at the IEEE Symposium on Haptic Interfaces for Virtual Environments and Teleoperator Systems, Reno, USA.

Huk, T. (2006). Who benefits from learning with 3D models? The case of spatial ability. Journal of Computer Assisted Learning, 22(6), 392-404.

Johansson, J. (2008). Efficient information visualization of multivariate and time-varying data, Linköping Studies in Science and Technology, Dissertations, No. 1191. Linköping: LiU-Tryck.

Jones, M. G., Minogue, J., Tretter, T. R., Negishi, A., \& Taylor, R. (2006). Haptic augmentation of science instruction: Does touch matter? Science Education, 90(1), 111123.

Kalyuga, S., Chandler, P., \& Sweller, J. (2004). When redundant on-screen text in multimedia technical instruction can interfere with learning. Human Factors, 46(3), 567-581.

Kozma, R. B. (1994). Will media influence learning? Reframing the debate. Educational Technology Research and Development, 42(2), 7-19.

Lederman, S. J., \& Klatzky, R. L. (1987). Hand movements: A window into haptic object recognition. Cognitive Psychology, 19(3), 342-368.

Martin, D., Eid, M., \& El Saddik, A. (2008). A haptic enabled DNA model sensing. Paper presented at the IEEE international symposium on parallel architectures, algorithms, and networks, Sydney, Australia.

Mayer, R. E., \& Moreno, R. (2002). Aids to computer-based multimedia learning. Learning and Instruction, 12(1), 107-119.

Mayer, R. E., \& Moreno, R. (2003). Nine ways to reduce cognitive load in multimedia learning. Educational Psychologist, 38(1), 43-52.

Minogue, J., \& Jones, M. G. (2006). Haptics in education: Exploring an untapped sensory modality. Review of Educational Research, 76(3), 317-348.

Minogue, J. Jones, M. G., Broadwell, B., \& Oppewall, T. (2006). The impact of haptic augmentation on middle school students' conceptions of the animal cell. Virtual Reality, 10(3-4), 293-305.

Moreno, R., \& Mayer, R. (2007). Interactive multimodal learning environments: Special issue on interactive learning environments: Contemporary issue and trends. Educational Psychology Review, 19(3), 309-326.

Reiner, M. (1999). Conceptual construction of fields through tactile interface. Interactive Learning Environments, 7(1), 31-55.

Richard, E., Tijou, A., Richard, P., \& Ferrier, J. -L. (2006). Multi-modal virtual environments for education with haptic and olfactory feedback. Virtual Reality, 10(3/4), 207-225.

Sathian, K., Zangaladze, A., Hoffman, J. M., \& Grafton, S. T. (1997). Feeling with the mind's eye. Neuroreport, 8(18), 3877-3881.

Schönborn, K. J., \& Anderson, T. R. (2009). A model of factors determining students' ability to interpret external representations in biochemistry. International Journal of Science Education, 31(2), 193-232.

Schönborn, K.J., \& Bögeholz, S. (2009). Knowledge transfer in biology and translation across external representations: Experts' views and challenges for learning. International Journal of Science and Mathematics Education, 7(5), 931-955.

Steinke, M., Huk, T., \& Floto, C. (2003). The use of high quality 3D animations and videos in hypermedia systems by learners with different cognitive abilities. In A. Rossett, Proceedings of world conference on e-learning in corporate, government, healthcare, and higher education (pp. 1193-1196). Chesapeake, VA: AACE. 
Tasker, R., \& Dalton, R. (2006). Research into practice: Visualisation of the molecular world using animations. Chemistry Education Research and Practice, 7(2), 141-159.

Tindall-Ford, S., Chandler, P., \& Sweller, J. (1997). When two sensory modes are better than one. Journal of Experimental Psychology: Applied, 3(4), 257-287.

Tsui, C. -Y., \& Treagust, D. F. (2007). Understanding genetics: Analysis of secondary students' conceptual status. Journal of Research in Science Teaching, 44(2), 205-235.

Wiebe, E. N., Minogue, J., Jones, M. G., Cowley, J., \& Krebs, D. (2009). Haptic feedback and students' learning about levers: Unraveling the effect of simulated touch. Computers \& Education, 53(3), 667-676.

Wilson, M. (2002). Six views of embodied cognition. Psychonomic Bulletin \& Review, 9(4), 625-636.

Wong, A., Marcus, N., Ayres, P., Smith, L., Cooper, G. A., Paas, F., \& Sweller, J. (2009). Instructional animations can be superior to statics when learning human motor skills. Computers in Human Behavior, 25(2), 339-347.

Wu, H. K., Krajcik, J. S., \& Soloway, E. (2001). Promoting understanding of chemical representations: Students' use of a visualization tool in the classroom. Journal of Research in Science Teaching, 38(7), 821-842. 